\title{
Role of astrocytes in manganese mediated neurotoxicity
}

\author{
Marta Sidoryk-Wegrzynowicz ${ }^{1}$ and Michael Aschner ${ }^{1,2^{*}}$
}

\begin{abstract}
Astrocytes are responsible for numerous aspects of metabolic support, nutrition, control of the ion and neurotransmitter environment in central nervous system (CNS). Failure by astrocytes to support essential neuronal metabolic requirements plays a fundamental role in the pathogenesis of brain injury and the ensuing neuronal death. Astrocyte-neuron interactions play a central role in brain homeostasis, in particular via neurotransmitter recycling functions. Disruption of the glutamine (Gln)/glutamate (Glu) - $\gamma$-aminobutyric acid (GABA) cycle (GGC) between astrocytes and neurons contributes to changes in Glu-ergic and/or GABA-ergic transmission, and is associated with several neuropathological conditions, including manganese (Mn) toxicity. In this review, we discuss recent advances in support of the important roles for astrocytes in normal as well as neuropathological conditions primarily those caused by exposure to Mn.
\end{abstract}

Keywords: Astrocytes, Manganese (Mn), Neurodegeneration, Transporter, Glutamine (Gln), Glutamate (Glu), Neurotransmission, PKC signaling, Ubiquitin-mediated proteolytic system

\section{Manganese: general characteristics and toxicity}

$\mathrm{Mn}$ is an essential trace metal, which is commonly found in the environment. Mn is present in all tissues and is required for the maintenance of proper function and regulation of numerous biochemical/cellular reactions [1]. $\mathrm{Mn}$ is an integral component of multiple enzymes, including glutamine synthetase (GS), mitochondrial superoxide dismutase (SOD), arginase and pyruvate carboxylae [2]. On the other hand, in humans excess deposition of $\mathrm{Mn}$ in the central nervous system (CNS) leads to neurological abnormalities, referred to as manganism [3]. Manganism is characterized by variety of psychiatric, cognitive and motor disturbances that resemble those inherent to Parkinson's disease (PD) [4]. However, the primary brain regions targeted by $\mathrm{Mn}$ are the globus pallidus and striatum of the basal ganglia, whereas neurodegeneration in PD is predominantly confined to the substantia nigra [5].

In the early stages of manganism, patients display psychotic symptoms, which progress to chronic disturbances in extrapyramidal circuits, leading to postural

\footnotetext{
* Correspondence: michael.aschner@vanderbilt.edu

'Department of Pediatrics, Vanderbilt University Medical Center, Nashville, TN 23233, USA

${ }^{2}$ Department of Pharmacology, the Kennedy Center for Research on Human Development, and the Center for Molecular Toxicology, Vanderbilt University Medical Center, Nashville, TN 23233, USA
}

instability, dystonia, bradyskinesia, micrographia, mask-like facial expression and speech disturbance [6-9]. Mn toxicity is a potential occupational health hazard in workers in ferroalloy plants, automotive repair technicians, battery manufacturers and welders [10-12]. Increased Mn levels in serum have been noted in chronic liver failure as a result of the inability to excrete the metal via the biliary system [13]. In addition, $\mathrm{Mn}$ pollution has been a subject of environmental concern because of consumption of contaminated water containing high levels of the metal as well as exposures from soy-based infant formulas and total parenteral nutrition [14]. Health risks associated with $\mathrm{Mn}$ exposure have also been associated with organic Mn-containing pesticides, such as $\mathrm{Mn}$ ethylenebis-dithiocarbamate [15]. An organic Mn compound methylcyclopentadienyl manganese tricarbonyl, a $\mathrm{Mn}$ derivative, is used as an antiknock agent in automobile fuels [16].

Mn transport within the CNS is mediated by several transporter proteins as a free ion or a non-specific protein-bound species [17]. In the $3^{+}$oxidation state $\mathrm{Mn}$ binds to the transferrin receptor (TfR) and its transport competes with iron (Fe) [18]. Transport of $\mathrm{Mn}$ in the $2^{+}$ oxidation state is mediated by the family of natural resistance-associated macrophage proteins (Nramp), the divalent metal transporter-1 (DMT-1) [19,20]. Moreover 
additional channels/transporters have been identified as Mn-transporting carriers. These include the divalent metal/bicarbonate ion symporters ZIP8 and ZIP14, various calcium channels, the solute carrier-39 (SLC39) family of zinc transporters, park9/ATP13A2, the magnesium $(\mathrm{Mg})$ transporter hip14 and the transient receptor potential melastatin 7 (TRPM7) [21], to name a few.

The mechanisms of Mn neurotoxicity are not completely understood. Oxidative/nitrosative stress (ONS) has been implicated in Mn-induced adverse effects [22,23]. Mn preferentially enters the mitochondrial matrix via the calcium $\left(\mathrm{Ca}^{2+}\right)$ uniporter [24]. It has slow clearance from mitochondria leading to increased matrix $\mathrm{Ca}^{2+}$ and reactive oxygen species (ROS) generation [25]. Mn also affects the antioxidant system by depleting glutathione (GSH) and glutathione peroxidase [26]. In addition, activation of oxidative stress-sensitive kinases and transcription factors, including nuclear factor- NF- $\mathrm{kB}$ has been implicated to mediate the neurotoxicity of $\mathrm{Mn}[27,28]$

\section{Astrocytes in the central nervous system function and dysfunction \\ General role of astrocytes in brain function: interaction with neurons}

Astrocytes are key regulators in brain function, characterized predominantly via their close interaction with neurons. Astrocyte metabolism, including energy generating pathways and amino acid homoeostasis is tightly coupled to that of neurons. This part of the review will discuss aspects of astrocyte metabolism which are involved in regulating key neuronal functions.

The metabolic relationship between astrocytes and neurons is critical for energy metabolism as well as for the synthesis of neurotransmitters [29]. Neurons are dependent upon astrocytes since they lack the enzyme pyruvate carboxylase (PC) and therefore dependent on astrocytes for de novo synthesis of glutamate (Glu) as well as for replenishment of Krebs cycle intermediates [30-32]. Moreover, Glu is not efficiently transported across the blood-brain barrier; thus astrocyte derived glucose serves as a precursor for synthesis of this neurotransmitter [33], and maintenance of optimal Glu levels require astrocytic support [34].

A major portion of astrocytic Gln is critical for maintaining Gln supply to Glu-ergic terminals as well as for generating neurotransmitters. Gln released from astrocytes via the bi-directional functioning amino acid systems $\mathrm{N}$ and ASC is taken up into neurons by the unidirectional system A [35,36]. In neurons Gln is metabolized to Glu, generating indirectly $\gamma$-aminobutyric acid (GABA) as well as the tricarboxylic acid (TCA) cycle intermediate, $\alpha$-ketoglutarate $(\alpha-K G)$ [29]. In turn, Glu released from neurons can be transported to the astrocyte via glutamate transporters [see below], where it is amidated to Gln, completing the metabolite shuttling between astrocyte and neurons, referred to as the glutamine/glutamateGABA cycle (GGC). Several enzymes which are necessary to ensure CNS glutamate and GABA homeostasis are heterogeneously distributed among neurons and astrocytes [37]. The glutamine synthesizing enzyme, GS, is exclusively localized in astrocytes [38], although few studies have reported its expression in both oligodendroglia and microglia under pathological conditions [39,40]. Studies in neuron-astrocyte co-cultures demonstrated that GS expression in the latter is positively regulated by neurons, and this effect appears to be mediated by neuron-derived Glu or trophic factors, requiring direct contact between astrocytes and nerve cell matrix [41,42]. An in situ study suggested that in the cerebellum phosphate-activated glutaminase (PAG) is primarily located in neurons rather than astrocytes [43], although a low activity of this enzyme has been observed in cultured astrocytes $[43,44]$.

The metabolic neuronal-astrocytic interaction is well demonstrated by the dependence of neurons on astrocytederived thiols for optimal maintenance of stable concentrations of GSH [45]. GSH, the main antioxidant constitutes $\sim 90 \%$ of the intracellular non-protein thiols and plays a prominent role in the detoxification of ROS and neutralization of organic hydroperoxides. It is noteworthy that GSH levels are lower in neurons than in astrocytes [46] and that cysteine derived from astrocytes is essential for the maintenance of stable GSH levels in neurons. In general, neuronal stores of GSH are largely dependent upon astrocytic stores, and neurons are more vulnerable than astrocytes to oxidative stress. Several co-culture studies have elegantly demonstrated that astrocytes protected neurons from toxicity by a GSH-dependent mechanism $[47,48]$.

Astrocytes also play a pivotal role in neurometabolic coupling, referred to as the astrocyte neuronal lactate shuttle hypothesis (ANLSH). ANLSH invokes that glucose enters the CNS via astrocytic processes [49]. ANLSH involves glutamate-stimulated aerobic glycolysis and uptake of Glu by astrocytes. The ensuing activation of the $\mathrm{Na}^{+}-\mathrm{K}^{+}$-ATPase triggers glucose uptake and processing via glycolysis, thus mediating release of lactate from astrocytes [50]. Lactate can be shuttled into neurons where it is used to meet their energy demand and works as a potent neuroprotective agent [51]. More recently, the alternative neuron-astrocyte lactate shuttle (NALS) hypothesis was developed, suggesting that depending on the thermodynamic and kinetic status of the cytosolic and mitochondrial redox states, lactate transfers from neurons to astrocytes [52]. In general, studies support the notion that neurons have the highest energy demand of all neural cells and are most vulnerable to energy failure [53]. It is important to note that recent modeling studies of astrocyte-neuron metabolic interactions have minimized the role of lactate 
as a major energy agent, suggesting that neurons are also capable of utilizing different fuel sources for incorporation into metabolic pathways, such as glucose, ketone bodies and fatty acids [54].

Intermediate filament proteins expressed in astrocytes, such as glial fibrillary acidic protein (GFAP) and vimentin, are involved in processes by which astrocytes control neurogenesis as well as other aspects of neural plasticity and regeneration. The ablation of GFAP and vimentin increases axonal and synaptic regeneration in mice [55-58]. Furthermore, thrombospondins extracellular matrix proteins secreted by astrocytes have been shown to promote synaptogenesis during development as well as in experimental mouse models of stroke [59].

It is noteworthy that astrocytes also play a dynamic role in CNS function by maintaining the restrictive properties of the blood-brain barrier (BBB). As an essential neurovascular component, astrocytes directly regulate the properties of this barrier. Furthermore, astrocytes can regulate the neurogenic niche indirectly by regulating the accessibility of blood-borne factors/molecules which are involved in neurogenesis [60].

Finally, astrocytes release factors that sustain neuronal function and viability. Astrocytes synthesize and secrete a wide range of neurotrophic and growth factors, cytokines, extracellular matrix proteins, proteoglycans and cholesterol which are involved in neuronal survival, proliferation, differentiation and synaptogenesis $[61,62]$.

\section{Astrocytes and manganese in some neuropathological conditions: focus on Alzheimer's disease (AD) and chronic hepatic encephalopathy (HE)}

Astrocytes play a critical role in the progression and outcome of neuropathological processes by reducing neural damage and promoting the revascularization of the surrounding tissue through reactive astrogliosis and neuroinflammation, the latter characterized by secretion of pro-inflammatory factors, such as interleukins [62-66]. On the other hand, reactive astrogliosis has been shown to be associated with functional impairment of astrocytes, and diminished neuronal support by astrocytes has been invoked in multiple neuropathological conditions. For example, in mice expressing human mutant Tau, a model of neurological disorders, an increase of GFAPimmunoreactive astrocytes and neuronal loss were shown in the spinal cord [67]. Other studies have implicated astrocyte-mediated Glu recycling in the pathogenesis of Alzheimer's disease (AD) [68]. Impairment in astrocytic Glu transport and reduction in the expression of the Glu transporters, L-glutamate-L-aspartate transporter (GLAST) and glutamate transporter 1 (GLT-1) has been invoked in a mouse model of Tau pathology. In homogenates of AD cortex, GFAP and GLAST expression were shown to inversely correlate with increased expression of GFAP and down regulation of GLAST as well as increased Braak stage [69]. Additionally, major disruption in Gln metabolism has been shown in AD. For example, GS activity in cortical homogenates from AD brain was found to be lower in comparison to homogenates derived from non-demented brains [70]. Moreover, the concentration of Glu was shown to be elevated, while the concentration of Gln was lower in cerebrospinal fluid of AD patients [71].

It is important to note that $\mathrm{Mn}$ was shown to induce cell swelling in cultured astrocytes and that astrocytic pathology, such as gliosis and Alzheimer type II astrocytes were observed in both animal and cell culture models in response to manganese exposures [72,73]. Morphologic changes of astrocytes are also a major feature of hepatocerebral disorders, including chronic hepatic encephalopathy (HE) [74]. Hepatic encephalopathy is a clinical complication of liver failure with a wide spectrum of neuropsychiatric complication. Glial cells were described as a primarily target in HE and as a cell responsible for the neuronal pathology [75]. The most prominent histopathological changes found in HE associated with chronic liver failure include Alzheimer's type II astrocytosis and astrocytic swelling, leading to brain edema [75-77]. Notably, Mn was found to be a significant etiologic factor in low-grade brain edema observed in $\mathrm{HE}$, affecting the dopaminergic neuronal system and dopaminergic receptor activity [73,78]. Interestingly, Mn and another important etiologic factor in $\mathrm{HE}$, namely ammonia, cause similar morphologic and functional changes in astrocytes. Both are potent glial toxins and have the capacity to act synergistically to activate the mitochondrial benzodiazepine receptor leading to increased synthesis of neurosteroids and GABAergic signaling [79]. Moreover Mn and ammonia downregulate the astrocytic Glu transporters, leading to impairment in neurotransmission [80].

\section{Manganese involvement in astrocyte function}

Brain Mn is preferentially deposited in astrocytes given the presence of high-capacity transporters within these cells. The concentration of $\mathrm{Mn}$ in astrocytes is 50-60 higher than in neurons [81]. At the subcellular level, the highest Mn concentration in astrocytes is noted within mitochondria [82]. Mn causes oxidative stress in primary cultures of astrocytes, leading to the mitochondrial dysfunction and energy insufficiency [22,83]. One of the possible mechanism of Mn-dependent failure of astrocytes to maintain antioxidant defence mechanisms is disruption of glutathione (GSH) synthesis [72,84,85]. In addition, Mn has a similar effect on taurine in both neurons and co-cultures; the latter also serves as a free radical scavenger and important neuroprotective amino acid [86].

Brain energy metabolism depends almost exclusively on the oxidation of glucose [87]. Glucose metabolism 
leads to the biosynthesis of neurotransmitters such as Glu, aspartate, and GABA [38]. Mn causes metabolic changes in astrocytic glucose metabolism by inhibition of the astrocyte-specific enzyme, GS [88,89]. Mn also plays an important role in brain energy metabolism by affecting the key anaplerotic, glial-specific enzyme, PC [90,91]. These Mn-induced effects on astrocytic pathology may cause a dyshomeostasis between neurons and astrocytes, leading to further neuronal synaptic dysfunction and activation of an excitotoxic state.

Mn was also shown to induce the expression of astrocytic inflammatory products as well as signal transduction mediators [72,92]. A recent study has shown increased transport of the nitric oxide (NO) substrate, L-arginine in Mn exposed astrocytes [93]. Other reports confirmed that $\mathrm{Mn}$ affects astrocytic inducible nitric oxide synthase (iNOS) expression and NO production upon activation of C6 glioma cells with LPS/ TNF $\gamma$ or primary astrocyte cultures with TNF $\alpha / \mathrm{TNF} \gamma[94,95]$. Astrocytic exposure to $\mathrm{Mn}$ is also associated with cell swelling secondary to NOS activation. Moreover, increased expression of the water channel protein aquaporin-4 (AQP4), a predominant astrocytic isoform, has been implicated in Mn-mediated cell swelling [96].

As mentioned above, astrocytes support multiple neuronal functions through multiple processes; thus Mn-mediated disturbances in astrocytes function would be expected to cause neuronal demise. Indeed, an in vivo study revealed that $\mathrm{Mn}$-mediated neuronal injury in the striatum and the globus pallidus is associated with primary dysfunction of astrocytes via mechanisms involving NO [94]. An In vitro study demonstrated that $\mathrm{Mn}$ inhibits the ability of astrocytes to promote neuronal differentiation by a mechanism that involves oxidative stress and a reduction in levels of the extracellular matrix protein, fibronectin [97].

\section{Glutamine in the central nervous system}

Gln abounds in the CNS, and its concentrations are at least one order of magnitude higher than any of the other amino acid in the interstitial and cerebrospinal fluid (CSF) [98]. Gln plays a prominent role in general CNS metabolism by supporting tissue homeostasis. As discussed above, Gln is the amino acid that directly couples ammonia metabolism to the synthesis of the amino acid neurotransmitter Glu, and indirectly to GABA. This reaction requires active communication between neurons and astrocytes, and is accomplished by the GGC cycle. The major role of the GGC is to thwart the extracellular Glu levels, thus preventing excitotoxicity. Moreover, Gln cycling between neurons and astrocytes produces compounds that are direct precursors of the tricarboxylic acid (TCA) cycle intermediate $\alpha$-ketoglutarate, thus maintaining the high demand for energy within the brain [29].
Transport of Gln across the membranes of CNS cells is mediated by multiple transport systems, characterized by their overlapping substrate specificities, substrate affinities and cellular distribution [36]. Among these systems, the sodium-dependent systems ASC, A and N play dominating roles in Gln turnover. Immunocytochemical analysis and Gln transport measurements both in vivo and in vitro revealed the compartmentation of Glntransporting proteins between astrocytes and neurons. The bi-directional system $\mathrm{N}$ transporters SNAT3 or SNAT5 which catalyze Gln transport, are exclusively located within astrocytes. SNAT3 is believed to specifically mediate Gln efflux from astrocytes [99,100]. In addition to System N, release of Gln from astrocytes is mediated by other transport systems, such as the sodium-independent System L (LAT2) and the sodium-dependent System ASC (ASCT2) [98,101,102]. The unidirectional system A transporter SNAT1, which catalyzes Gln uptake, is predominately expressed in neurons [98]. A portion of astroglial-derived Gln across the BBB to the periphery is mediated by system $\mathrm{N}$ transporters along with some additional contribution from the System L transporters [103].

\section{Manganese and glutamine turnover}

The Gln/Glu-GABA cycle represents a complex process, since Gln efflux from astrocytes must be met by its influx in neurons. Mn toxicity is associated with the disruption of both of these critical points in the GGC. In cultured astrocytes, pre-treatment with $\mathrm{Mn}$ inhibits the initial net uptake of Gln in a concentration-dependent manner [83]. Mn added directly to astrocytes induces deregulation in the expression of SNAT3, SNAT2, ASCT2 and LAT2 transporters [83]. Corroborating the changes in transporter protein expression levels, astrocytes treated with $\mathrm{Mn}$ displayed a significant decrease in Gln uptake mediated by the transporting Systems N and ASC, and a decrease in Gln efflux mediated by Systems N, ASC and L (Table 1) [104].

The contribution of PKC signalling to Mn-induced dyshomeostasis in Gln transport has been investigated in cultured astrocytes. A recent study revealed that PKC inhibition by its general inhibitor bisindolylmaleimide II (BIS II) effectively reversed the Mn-induced downregulation in Glu uptake. Treatment of primary astrocyte cultures with a PKC stimulator caused decreased Gln uptake mediated by Systems ASC and N, and decreased expression of ASCT2 and SNAT3 protein levels in cell lysates and in plasma membranes [105]. It is noteworthy that both transporters contain putative PKC phosphorylation sites, conserved in the human, rat and mouse $[106,107]$. In addition, a recent in situ study showed that PKC activation induced phosphorylation and internalization of SNAT3 [108]. Furthermore, increased binding of PKC $\delta$ to ASCT2 and SNAT3 upon exposure 
Table 1 Manganese involvement in glutamine/glutamate-GABA cycle: disruption of glutamine and glutamate transporters

\begin{tabular}{|c|c|c|c|c|}
\hline Name of transporter & & Changes down (down-regulation) mediated by manganese & Cellular localization & References \\
\hline Glutamine transporter & System & & & \\
\hline SNAT3 & System N & mRNA and protein expression; function(uptake and efflux) & astrocytes & [67], [81] \\
\hline SNAT2 & System A & mRNA and protein expression & astrocytes & [81] \\
\hline ASCT2 & System ASC & protein expression; function (uptake and efflux) & astrocytes & [81] \\
\hline LAT2 & System L & mRNA and protein expression; function (uptake) & astrocytes endothelial cells & [81] \\
\hline \multicolumn{5}{|l|}{ Glutamine transporter } \\
\hline GLAST & & protein expression; function (uptake) & astrocytes & [98], [103] \\
\hline GLT-1 & & mRNA and protein expression; function (uptake) & astrocytes & [98], [103] \\
\hline
\end{tabular}

to $\mathrm{Mn}$ has been identified by co-immunoprecipitation. In contrast, Mn exposure causes increased phosphorylation of PKC $\delta$ in cultured astrocytes. Taken together, these findings suggest a prominent role of $\mathrm{PKC} \delta$ in $\mathrm{Mn}$-mediated disruption of Gln turnover (Figure 1).

\section{Ubiquitin-mediated proteolytic system in Mn-mediated disruption of SNAT3}

A recent study revealed that the astrocytic transporter SNAT3 possesses the highest affinity/specificity to Gln among of all the investigated carriers, and that it is the most sensitive to Mn exposure. This transporter is readily degraded subsequent to a relatively short exposure to $\mathrm{Mn}$ [104]. In addition, it was suggested that the ubiquitin-mediated proteolytic system might be involved in the Mn-mediated down-regulation of SNAT3 [105]. $\mathrm{Mn}$ exposure has been noted to increase both free ubiquitin levels and ubiquitinated proteins in primary cultures of astrocytes. Furthermore, we recently showed a selective interaction of SNAT3 with the ubiquitin ligase, Nedd4-2

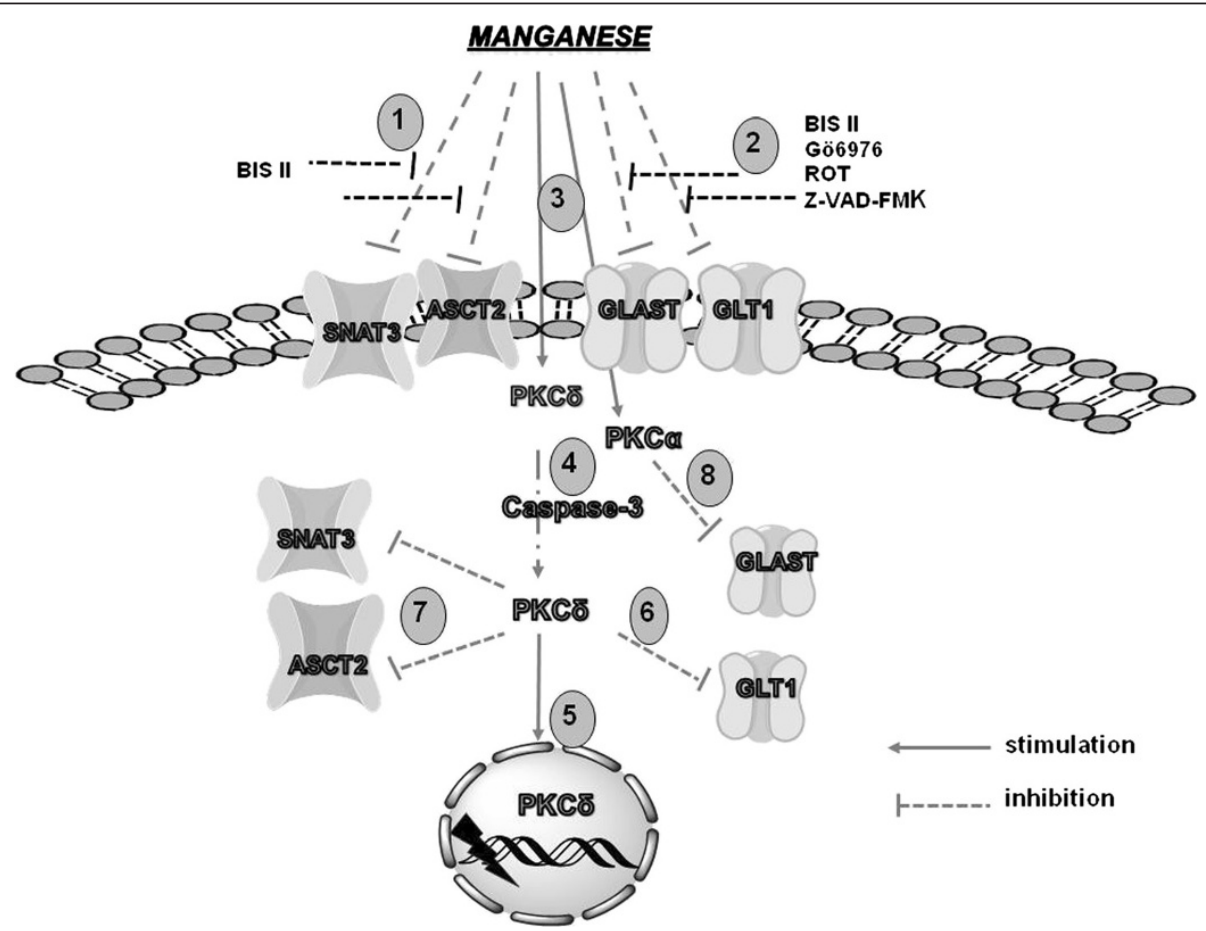

Figure 1 A proposed model describing the mechanisms of Mn-mediated disruption of glutamine/glutamate-GABA cycle. Mn exposure leads to the deregulation of Gln uptake by dysfunction of SNAT3 and ASCT2 activity, while incubation with BIS II (bisindolylmaleimide, general PKC inhibitor) reverses this effect (1); Mn exposure leads to the deregulation of Glu uptake by dysfunction of GLT1 and GLAST activity, while incubation with BIS II, Gö6976 (PKCa inhibitor) , ROT (rottlerin, PKC inhibitor) and Z-VAD-FMK (Z-Ala-Glu(OMe)-Val-Asp(OMe)-fluoromethyl ketone caspase inhibitor) reverses this effect (2); Mn exposure leads to the activation of PKC $\delta$ and PKCa (3); additionally, Mn mediates activation of PKC $\delta$ by caspase-3-dependent proteolytic cleavage (4); proteolytically activated PKC $\delta$ translocates to the nucleus and mediates DNA fragmentation (5); Mn-mediated disruption of SNAT3, ASCT2 and GLT1 expression involves PKCS signalling (6,7); GLAST expression is down regulated by Mn via PKCa signalling (8). 
(neuronal precursor cell expressed, developmentally down-regulated 4-2) upon Mn exposure in astrocytes [105]. It is noteworthy that Nedd4-2 mediates the down-regulation of numerous membrane channels and transporters via ubiquitination and subsequent degradation by the proteasome [109]. Polyubiquitination plays a key role in transporter sorting and targeting for lysosomal degradation, which also involves the proteasome [110]. A recent study has confirmed that serum- and glucocorticoid-induced kinase 1 (SGK1) phosphorylates the ubiquitin ligase Nedd4-2, decreasing the interaction between Nedd4-2 and target proteins [111]. Furthermore, in a Xenopus laevis oocyte expression system it has been suggested that SNAT3 is down regulated by Nedd4-2, and that this effect is restored by SGK1 [112]. Notably, Mn exposure leads to decreased SGK1 expression and phosphorylation, suggesting Nedd4-2/SGK1 involvement in Mn-mediated degradation of SNAT3. These findings, concomitant with evidence that chronic Mn exposure alters the expression of genes associated with the ubiquitin/proteasome system [113], suggest that $\mathrm{Mn}$ disrupts SNAT3 expression or function by promoting its ubiquitination [105].

It has also been shown that PKC activation results in the ubiquitination of the dopamine transporter (DAT), leading to its internalization and degradation $[114,115]$. Furthermore, the knockdown of Nedd4-2 resulted in a dramatic reduction in the PKC-dependent ubiquitination of DAT [115]. PKC activation also led to hyper-ubiquitination, and increased the interaction between SNAT3 and Nedd4-2. PKC stimulation caused a decrease in astrocytic Gln uptake mediated by System N and significantly decreased SNAT3 protein levels, while Mn exposure activated PKCs [82]. Taken together, these findings demonstrate that Mn-induced deregulation of SNAT3 function is likely mediated via PKC signalling and accompanied by an increase in ubiquitin-mediated proteolysis.

\section{Manganese and glutamate transport}

Glu is the prominent excitatory neurotransmitter in the mammalian CNS [116]. Regulation of synaptic Glu concentrations is critical to normal CNS function. Glu released into the synaptic cleft in taken up by Glu transporters mainly present perisynaptically on astrocytes. Only a small amount is taken up by presynaptic neurons. Astrocytes take up glutamate via the sodium-dependent Glu transporters, GLAST, GLT-1 and the sodium-independent chloride-dependent Glu-cystine antiporter [29]. Accordingly, astrocytes regulate the levels of extracellular Glu and influence synaptic activity. Using antisense knockdown or pharmacological inhibition of Glu transporters, it has been demonstrated that disruption of transporter function increases the vulnerability of neurons to excitotoxic insults [31]. The functional relevance of the Glu transporters was demonstrated in animal studies, where knockout of GLAST resulted in impaired performance on an accelerating rotarod, and partial loss of GLT-1 led to hind limb paralysis $[117,118]$. Impairment in astrocyte-mediated recycling of Glu represents the major contributing factor to neuropathology, and it has been invoked in the etiology of several neurodegenerative diseases, including $\mathrm{AD}$, Parkinson disease (PD) and amyotrophic lateral sclerosis (ALS) [119].

Several studies established that Mn disrupts Glu transporting systems leading to both a reduction in Glu uptake and elevation in extracellular Glu levels [120] (Table 1). For instance, Chinese hamster ovary $(\mathrm{CHO})$ cells transfected with GLAST or GLT-1 show impairment in Glu transport in response to Mn exposure [121]. Longterm airborne $\mathrm{Mn}$ exposure leads to the down regulation of GLAST and GLT-1 In non-human primate brain, both at the mRNA and protein levels [84]. Although the mechanisms of $\mathrm{Mn}$-mediated disruption in transporter expression have yet to be completely understood, lysosomal proteolysis has been implicated in GLT-1 degradation upon exposure to this metal $[122,123]$.

PKC signalling has been implicated in $\mathrm{Mn}$-induced down-regulation of Glu turnover in primary astrocyte cultures [124]. A recent study revealed that PKC stimulation by $\alpha$-phorbol 12 -myristate (PMA) significantly decreased astrocytic Glu uptake, while treatment with the general PKC inhibitor BIS II, reversed the Mn-induced down regulation of Glu transport. Moreover, Mn-dependent down-regulation in astrocytic Glu uptake was reversed by specific inhibitors of PKC $\delta$ - rottlerin (ROT), and PKC $\alpha$ - Gö6976 or caspase 3 inhibitorZ-Ala-Glu(OMe)-Val-Asp(OMe)-fluoromethyl ketone (Z-VAD-FMK) [122]. Similarly, Mn-induced downregulation of GLT1 protein level was reversed by Bis II, ROT, Gö6976 and Z-VAD-FMK inhibitors. Mn-dependent down-regulation of astrocytic GLAST expression was also attenuated in the presence of PKC $\alpha$ and casapase-3 inhibitors. Furthermore, direct association between GLT-1, but no GLAST, and the PKC $\delta$ or PKC $\alpha$ isoforms, and Mn-induced increases in PKC $\delta$-GLT-1 interaction have been noted in a co-immunoprecipitation study [122].

The role of the PKC $\delta$ isoform in Mn-induced deregulation of Glu turnover was estimated by a knockdown study. Astrocytes transfected with shRNA against PKC $\delta$, (but not with $\mathrm{PKC} \alpha$ ) showed lessened sensitivity to $\mathrm{Mn}$ compared to those transfected with control shRNA, suggesting a predominant role for the PKC $\delta$ isoform in Mn-dependent down-regulation of Glu turnover [124]. Parallel to these observations, recent evidence shows that PKC signalling is critical in Glu transporter regulation [104]. PKC-mediated decrease in Glu transporter function may involve changes in transporter activity or number/ expression by increasing the rates of endocytosis or decreasing the redistribution of the transporters from 
a subcellular compartment to the plasma membrane. Another possible mechanism of PKC-induced negative regulation of Glu transporters invokes down-regulation of transporter activity at the cell membrane. For example, in C6 glioma cells transfected with GLT-1 and in primary cultures endogenously expressing GLT-1, activation of PKC rapidly down regulates GLT-1 cell surface expression [124]. As far as GLAST transporter function, several groups found contradictory results, showing decreases as well as increases in GLAST function in response PKC activation [125]. Several studies using cell cultures as well as animal models indicate that proteolytic activation of PKC $\delta$ by caspase- 3 plays a major role in the regulation and execution of apoptosis [126]. In addition, previous studies showed that caspase-3-dependent PKC $\delta$ activation not only contributes to neuronal apoptosis, but also has a regulatory role in amplification of the apoptotic cascade during neurotoxic stress inherent to $\mathrm{Mn}$ treatment via positive feedback loop between PKC $\delta$ and caspase-3 activation [127]. Proteolytic activation of $\mathrm{PKC} \delta$ promotes its nuclear translocation and PKC $\delta$-dependent mediation of DNA fragmentation. Notably, Mn toxicity is associated with caspase-3 activation in several in vitro and in vivo models [127-130] as well as increased PKC $\delta$ 's nuclear translocation [124]. Cotreatment with the PKC $\delta$ inhibitor ROT or the caspase3 inhibitor Z-DEVD-FMK blocked Mn-induced DNA fragmentation in mesencephalic dopaminergic neuronal [N27] cells. Additionally, N27 cells expressing a catalytically inactive $\mathrm{PKC} \delta$ protein or a caspase-3 cleavage resistant PKC $\delta$ protein were found to be resistant to Mn-mediated apoptosis [127].

A mechanism implicating caspase-3 and PKC $\delta$ inhibition has been invoked for the Mn-mediated disruption of Glu and Gln transport in astrocytes [124]. Collectively, activation of PKC signalling seems to be a ubiquitous mechanism for the regulation of GGC by Mn (Figure 1).

\section{Review and Conclusion}

Astrocytes are involved at multiple levels in brain (patho) physiology via their interaction with neurons. The role of astrocytes in neuronal activity and survival is well illustrated in pathological conditions mediated by disruption of the Gln/Glu-GABA cycle. The deregulation of Gln homeostasis may consequently diminish the availability of this amino acid to neurons. These effects are posited to lead to impairment of Glu-ergic neurotransmission and are very well recognized in Mn neurotoxicity. The findings reported in this review suggest that a better understanding of Gln cycling between neurons and astrocytes may provide knowledge about normal brain function and highlight potential molecular tools for therapeutic interventions in pathology caused by Mn.

\section{Competing interests}

The authors declare that they have no competing interests.

\section{Authors' contributions}

MSW carried out several of the studies that are reviewed herein and was the lead author, synthesized the literature and was involved in drafting the manuscript. MA provide conceptual input and participated in the coordination. All authors read and approved the final manuscript.

\section{Acknowledgements}

The authors wish to acknowledge partial support by grants from the National Institute of Environmental Health Sciences (NIEHS) ES R01 10563 and ES P30 000267.

Received: 13 December 2012 Accepted: 10 April 2013

Published: 18 April 2013

\section{References}

1. Keen CL, Ensunsa JL, Clegg MS: Manganese metabolism in animals and humans including the toxicity of manganese. Met lons Biol Syst 2000, 37:89-121.

2. Wedler FC, Denman RB: Glutamine synthetase: the major Mn (II) enzyme in mammalian brain. Curr Top Cell Regul 1984, 24:153-169.

3. Erikson KM, Syversen T, Aschner JL, Aschner M: Interactions between excessive manganese exposures and dietary iron-deficiency in neurodegeneration. Environ Toxicol Pharmacol 2005, 19(3):415-421.

4. Olanow CW: Manganese-induced parkinsonism and Parkinson's disease. Ann N Y Acad Sci 2004, 1012:209-223.

5. Lucchini RG, Martin CJ, Doney BC: From manganism to manganeseinduced parkinsonism: a conceptual model based on the evolution of exposure. Neuromolecular Med 2009, 11(4):311-321.

6. Mergler D, Baldwin M: Early manifestations of manganese neurotoxicity in humans: an update. Environ Res 1997, 73(1-2):92-100.

7. Olanow CW, Good PF, Shinotoh H, Hewitt KA, Vingerhoets F, Snow BJ, Beal MF, Calne DB, Perl DP: Manganese intoxication in the rhesus monkey: a clinical, imaging, pathologic, and biochemical study. Neurology 1996, 46(2):492-498.

8. Kim Y, Bowler RM, Abdelouahab N, Harris M, Gocheva V, Roels HA: Motor function in adults of an Ohio community with environmental manganese exposure. NeuroToxicology 2011, 32(5):606-614.

9. Bowler RM, Gysens S, Diamond E, Nakagawa S, Drezgic M, Roels HA: Manganese exposure: neuropsychological and neurological symptoms and effects in welders. NeuroToxicology 2006, 27(3):315-326.

10. Lander F, Kristiansen J, Lauritsen JM: Manganese exposure in foundry furnacemen and scrap recycling workers. Int Arch Occup Environ Health 1999, 72(8):546-550.

11. Lucchini R, Bergamaschi E, Smargiassi A, Festa D, Apostoli P: Motor function, olfactory threshold, and hematological indices in manganese-exposed ferroalloy workers. Environ Res 1997, 73(1-2):175-180.

12. Wang D, Du X, Zheng W: Alteration of saliva and serum concentrations of manganese, copper, zinc, cadmium and lead among career welders. Toxicol Lett 2008, 176(1):40-47.

13. McKinney AM, Filice RW, Teksam M, Casey S, Truwit C, Clark HB, Woon C, Liu HY: Diffusion abnormalities of the globi pallidi in manganese neurotoxicity. Neuroradiology 2004, 46(4):291-295.

14. Krachler M, Rossipal E: Concentrations of trace elements in extensively hydrolysed infant formulae and their estimated daily intakes. Ann Nutr Metab 2000, 44(2):68-74.

15. Ferraz HB, Bertolucci PH, Pereira JS, Lima JG, Andrade LA: Chronic exposure to the fungicide maneb may produce symptoms and signs of CNS manganese intoxication. Neurology 1988, 38(4):550-553.

16. Finkelstein $M M$, Jerrett $M: A$ study of the relationships between Parkinson's disease and markers of traffic-derived and environmental manganese air pollution in two Canadian cities. Environ Res 2007, 104(3):420-432.

17. Aschner $M$, Gannon M: Manganese $(\mathrm{Mn})$ transport across the rat blood-brain barrier: saturable and transferrin-dependent transport mechanisms. Brain Res Bull 1994, 33(3):345-349.

18. Aschner M, Aschner JL: Manganese neurotoxicity: cellular effects and blood-brain barrier transport. Neurosci Biobehav Rev 1991, 15(3):333-340.

19. Aschner M, Vrana KE, Zheng W: Manganese uptake and distribution in the central nervous system (CNS). NeuroToxicology 1999, 20(2-3):173-180.

20. Fitsanakis VA, Au C, Erikson KM, Aschner M: The effects of manganese on glutamate, dopamine and gamma-aminobutyric acid regulation. Neurochem Int 2006, 48(6-7):426-433. 
21. Bowman AB, Kwakye GF, Hernandez EH, Aschner M: Role of manganese in neurodegenerative diseases. J Trace Elem Med Biol Organ Soc Mineral Trace Elem 2011, 25(4):191-203

22. Chen CJ, Liao SL: Oxidative stress involves in astrocytic alterations induced by manganese. Exp Neurol 2002, 175(1):216-225.

23. Erikson KM, Dobson AW, Dorman DC, Aschner M: Manganese exposure and induced oxidative stress in the rat brain. Sci Total Environ 2004 334-335:409-416.

24. Gavin CE, Gunter KK, Gunter TE: Manganese and calcium efflux kinetics in brain mitochondria, Relevance to manganese toxicity. Biochem J 1990, 266(2):329-334.

25. Kowaltowski AJ, Castilho RF, Vercesi AE: Ca (2+)-induced mitochondrial membrane permeabilization: role of coenzyme Q redox state. Am J Physiol 1995, 269(1 Pt 1):C141-147.

26. Liccione JJ, Maines MD: Selective vulnerability of glutathione metabolism and cellular defense mechanisms in rat striatum to manganese. J Pharmacol Exp Ther 1988, 247(1):156-161.

27. Prabhakaran K, Ghosh D, Chapman GD, Gunasekar PG: Molecular mechanism of manganese exposure-induced dopaminergic toxicity. Brain Res Bull 2008, 76(4):361-367.

28. Yin Z, Aschner JL, dos Santos AP, Aschner M: Mitochondrial-dependent manganese neurotoxicity in rat primary astrocyte cultures. Brain Res 2008, 1203:1-11.

29. Bak LK, Schousboe A, Waagepetersen HS: The glutamate/GABA-glutamine cycle: aspects of transport, neurotransmitter homeostasis and ammonia transfer. J Neurochem 2006, 98(3):641-653.

30. Yu AC, Drejer J, Hertz L, Schousboe A: Pyruvate carboxylase activity in primary cultures of astrocytes and neurons. J Neurochem 1983, 41(5): 1484-1487.

31. Danbolt NC: Glutamate uptake. Prog Neurobiol 2001, 65(1):1-105

32. Hertz L, Zielke HR: Astrocytic control of glutamatergic activity: astrocytes as stars of the show. Trends Neurosci 2004, 27(12):735-743.

33. Hertz L, Dienel GA: Energy metabolism in the brain. Int Rev Neurobiol 2002, 51:1-102.

34. Hertz L: Glutamate, a neurotransmitter-and so much more, A synopsis of Wierzba III. Neurochem Int 2006, 48(6-7):416-425.

35. Tamarappoo BK, Raizada MK, Kilberg MS: Identification of a system N-like $\mathrm{Na}(+)$-dependent glutamine transport activity in rat brain neurons. J Neurochem 1997, 68(3):954-960.

36. Collarini EJ, Oxender DL: Mechanisms of transport of amino acids across membranes. Annu Rev Nutr 1987, 7:75-90.

37. Hertz L, Dringen R, Schousboe A, Robinson SR: Astrocytes: glutamate producers for neurons. J Neurosci Res 1999, 57(4):417-428.

38. Norenberg MD, Martinez-Hernandez A: Fine structural localization of glutamine synthetase in astrocytes of rat brain. Brain Res 1979, 161(2):303-310.

39. Gras G, Samah B, Hubert A, Leone C, Porcheray F, Rimaniol AC: EAAT expression by macrophages and microglia: still more questions than answers. Amino Acids 2012, 42(1):221-229.

40. Takasaki C, Yamasaki M, Uchigashima M, Konno K, Yanagawa Y, Watanabe M: Cytochemical and cytological properties of perineuronal oligodendrocytes in the mouse cortex. Eur J Neurosci 2010, 32(8):1326-1336.

41. Derouiche A, Frotscher M: Astroglial processes around identified glutamatergic synapses contain glutamine synthetase: evidence for transmitter degradation. Brain Res 1991, 552(2):346-350.

42. Westergaard N, Larsson OM, Jensen B, Schousboe A: Synthesis and release of GABA in cerebral cortical neurons co-cultured with astrocytes from cerebral cortex or cerebellum. Neurochem Int 1992, 20(4):567-575.

43. Kvamme E, Svenneby G, Hertz L, Schousboe A: Properties of phosphate activated glutaminase in astrocytes cultured from mouse brain. Neurochem Res 1982, 7(6):761-770.

44. Schousboe A, Hertz L, Svenneby G, Kvamme E: Phosphate activated glutaminase activity and glutamine uptake in primary cultures of astrocytes. J Neurochem 1979, 32(3):943-950.

45. Dringen R, Hirrlinger J: Glutathione pathways in the brain. Biol Chem 2003, 384(4):505-516.

46. Sagara JI, Miura K, Bannai S: Maintenance of neuronal glutathione by glial cells. J Neurochem 1993, 61(5):1672-1676.

47. Dringen R, Pfeiffer B, Hamprecht B: Synthesis of the antioxidant glutathione in neurons: supply by astrocytes of CysGly as precursor for neuronal glutathione. J Neurosci Offic J Soc Neurosci 1999, 19(2):562-569.
48. Wang XF, Cynader MS: Astrocytes provide cysteine to neurons by releasing glutathione. J Neurochem 2000, 74(4):1434-1442.

49. Pellerin L, Magistretti PJ: Neuroenergetics: calling upon astrocytes to satisfy hungry neurons. Neurosci Rev J Bringing Neurobiol Neurol Psychiatr 2004, 10(1):53-62.

50. Occhipinti R, Somersalo E, Calvetti D: Astrocytes as the glucose shunt for glutamatergic neurons at high activity: an in silico study. J Neurophysiol 2009, 101(5):2528-2538.

51. Belanger M, Magistretti PJ: The role of astroglia in neuroprotection. Dialogues Clin Neurosci 2009, 11(3):281-295.

52. Simpson IA, Carruthers A, Vannucci SJ: Supply and demand in cerebral energy metabolism: the role of nutrient transporters. J Cereb Blood Flow Metab Offic J Int Soc Cereb Blood Flow Metabol 2007, 27(11):1766-1791.

53. Nehlig A, Coles JA: Cellular pathways of energy metabolism in the brain: is glucose used by neurons or astrocytes? Glia 2007, 55(12):1238-1250.

54. Mangia S, DiNuzzo M, Giove F, Carruthers A, Simpson IA, Vannucci SJ: Response to 'comment on recent modeling studies of astrocyte-neuron metabolic interactions': much ado about nothing. J Cereb Blood Flow Metabol Offic J Int Soc Cereb Blood Flow Metabol 2011, 31(6):1346-1353.

55. Colucci-Guyon E, Gimenez YRM, Maurice T, Babinet C, Privat A: Cerebellar defect and impaired motor coordination in mice lacking vimentin. Glia 1999, 25(1):33-43.

56. Pekny M, Nilsson M: Astrocyte activation and reactive gliosis. Glia 2005, 50(4):427-434

57. Wilhelmsson U, Li L, Pekna M, Berthold CH, Blom S, Eliasson C, Renner O, Bushong E, Ellisman M, Morgan TE, et al: Absence of glial fibrillary acidic protein and vimentin prevents hypertrophy of astrocytic processes and improves post-traumatic regeneration. J Neurosci Offic J Soc Neurosci 2004, 24(21):5016-5021.

58. Colucci-Guyon E, Portier MM, Dunia I, Paulin D, Pournin S, Babinet C: Mice lacking vimentin develop and reproduce without an obvious phenotype. Cell 1994, 79(4):679-694.

59. Liauw J, Hoang S, Choi M, Eroglu C, Choi M, Sun GH, Percy M, WildmanTobriner B, Bliss T, Guzman RG, et al: Thrombospondins 1 and 2 are necessary for synaptic plasticity and functional recovery after stroke. J Cereb Blood Flow Metabol Offic J Int Soc Cereb Blood Flow Metabol 2008, 28(10):1722-1732.

60. Liebner S, Czupalla CJ, Wolburg H: Current concepts of blood-brain barrier development. Int J Dev Biol 2011, 55(4-5):467-476.

61. Farina C, Aloisi F, Meinl E: Astrocytes are active players in cerebral innate immunity. Trends Immunol 2007, 28(3):138-145.

62. Blondel O, Collin C, McCarran WJ, Zhu S, Zamostiano R, Gozes I, Brenneman $\mathrm{DE}$, McKay RD: A glia-derived signal regulating neuronal differentiation. J Neurosci Offic J Soc Neurosci 2000, 20(21):8012-8020.

63. Heneka MT, Rodriguez JJ, Verkhratsky A: Neuroglia in neurodegeneration. Brain Res Rev 2010, 63(1-2):189-211.

64. Verkhratsky A, Olabarria M, Noristani HN, Yeh CY, Rodriguez JJ: Astrocytes in Alzheimer's disease. Neurotherapeutics J Am Soc Exp NeuroTherapeutics 2010, 7(4):399-412

65. Samland H, Huitron-Resendiz S, Masliah E, Criado J, Henriksen SJ, Campbell IL: Profound increase in sensitivity to glutamatergic- but not cholinergic agonist-induced seizures in transgenic mice with astrocyte production of IL-6. J Neurosci Res 2003, 73(2):176-187.

66. Aloisi F, Care A, Borsellino G, Gallo P, Rosa S, Bassani A, Cabibbo A, Testa U, Levi G, Peschle C: Production of hemolymphopoietic cytokines (IL-6, IL-8, colony-stimulating factors) by normal human astrocytes in response to IL-1 beta and tumor necrosis factor-alpha. J Immunol 1992, 149(7):2358-2366.

67. Yata K, Oikawa S, Sasaki R, Shindo A, Yang R, Murata M, Kanamaru K, Tomimoto H: Astrocytic neuroprotection through induction of cytoprotective molecules; a proteomic analysis of mutant P301S tau-transgenic mouse. Brain Res 2011, 1410:12-23.

68. Maragakis NJ, Rothstein JD: Glutamate transporters in neurologic disease. Arch Neurol 2001, 58(3):365-370.

69. Simpson JE, Ince PG, Lace G, Forster G, Shaw PJ, Matthews F, Savva G, Brayne C, Wharton SB, Function MRCC, et al: Astrocyte phenotype in relation to Alzheimer-type pathology in the ageing brain. Neurobiol Aging 2010, 31(4):578-590.

70. Le Prince G, Delaere P, Fages C, Lefrancois T, Touret M, Salanon M, Tardy M: Glutamine synthetase (GS) expression is reduced in senile dementia of the Alzheimer type. Neurochem Res 1995, 20(7):859-862. 
71. Redjems-Bennani N, Jeandel C, Lefebvre E, Blain H, Vidailhet M, Gueant JL: Abnormal substrate levels that depend upon mitochondrial function in cerebrospinal fluid from Alzheimer patients. Gerontology 1998, 44(5):300-304

72. Rama Rao KV, Reddy PV, Hazell AS, Norenberg MD: Manganese induces cell swelling in cultured astrocytes. NeuroToxicol 2007, 28(4):807-812.

73. Hazell AS, Normandin L, Norenberg MD, Kennedy G, Yi JH: Alzheimer type II astrocytic changes following sub-acute exposure to manganese and its prevention by antioxidant treatment. Neurosci Lett 2006, 396(3):167-171.

74. Hazell AS, Butterworth RF: Hepatic encephalopathy: An update of pathophysiologic mechanisms. Proc Soc Exp Biol Med Soc Exp Biol Med 1999, 222(2):99-112.

75. Norenberg MD: A light and electron microscopic study of experimental portal-systemic (ammonia) encephalopathy. Progression and reversal of the disorder. Lab Investig J Tech Meth Pathol 1977, 36(6):618-627.

76. Pilbeam CM, Anderson RM, Bhathal PS: The brain in experimental portal-systemic encephalopathy. I. Morphological changes in three animal models. J Pathol 1983, 140(4):331-345.

77. Traber PG, Dal Canto M, Ganger DR, Blei AT: Electron microscopic evaluation of brain edema in rabbits with galactosamine-induced fulminant hepatic failure: ultrastructure and integrity of the blood-brain barrier. Hepatology 1987, 7(6):1272-1277.

78. Butterworth RF: Neurotransmitter dysfunction in hepatic encephalopathy: new approaches and new findings. Metab Brain Dis 2001, 16(1-2):55-65.

79. Butterworth RF: Pathophysiology of hepatic encephalopathy: The concept of synergism. Hepatol Res Offic J Jpn Soc Hepatol 2008 38(Suppl 1):S116-121.

80. Jayakumar AR, Rama Rao KV, Kalaiselvi P, Norenberg MD: Combined effects of ammonia and manganese on astrocytes in culture. Neurochem Res 2004, 29(11):2051-2056.

81. Aschner M, Gannon M, Kimelberg HK: Manganese uptake and efflux in cultured rat astrocytes. J Neurochem 1992, 58(2):730-735.

82. Morello M, Canini A, Mattioli P, Sorge RP, Alimonti A, Bocca B, Forte G, Martorana A, Bernardi G, Sancesario G: Sub-cellular localization of manganese in the basal ganglia of normal and manganese-treated rats An electron spectroscopy imaging and electron energy-loss spectroscopy study. NeuroToxicology 2008, 29(1):60-72.

83. Milatovic D, Yin Z, Gupta RC, Sidoryk M, Albrecht J, Aschner JL, Aschner M: Manganese induces oxidative impairment in cultured rat astrocytes. Toxicol Sci Offic J Soc Toxicol 2007, 98(1):198-205.

84. Erikson KM, Dorman DC, Lash LH, Aschner M: Duration of airbornemanganese exposure in rhesus monkeys is associated with brain regional changes in biomarkers of neurotoxicity. NeuroToxicology 2008 29(3):377-385.

85. Dukhande W, Malthankar-Phatak GH, Hugus JJ, Daniels CK, Lai JC: Manganese-induced neurotoxicity is differentially enhanced by glutathione depletion in astrocytoma and neuroblastoma cells. Neurochem Res 2006, 31(11):1349-1357.

86. Saransaari P, Oja SS: Taurine and neural cell damage. Amino Acids 2000 19(3-4):509-526.

87. Gjedde A, Marrett S: Glycolysis in neurons, not astrocytes, delays oxidative metabolism of human visual cortex during sustained checkerboard stimulation in vivo. J Cereb Blood Flow Metab Offic J Int Soc Cereb Blood Flow Metab 2001, 21(12):1384-1392.

88. Erikson KM, Dorman DC, Fitsanakis V, Lash LH, Aschner M: Alterations of oxidative stress biomarkers due to in utero and neonatal exposures of airborne manganese. Biol Trace Elem Res 2006, 111(1-3):199-215.

89. Erikson KM, Dorman DC, Lash LH, Aschner M: Manganese inhalation by rhesus monkeys is associated with brain regional changes in biomarkers of neurotoxicity. Toxicol Sci Offic J Soc Toxicol 2007, 97(2):459-466.

90. Shank RP, Bennett GS, Freytag SO, Campbell GL: Pyruvate carboxylase: an astrocyte-specific enzyme implicated in the replenishment of amino acid neurotransmitter pools. Brain Res 1985, 329(1-2):364-367.

91. Zwingmann C, Leibfritz D, Hazell AS: Energy metabolism in astrocytes and neurons treated with manganese: relation among cell-specific energy failure, glucose metabolism, and intercellular trafficking using multinuclear NMR-spectroscopic analysis. J Cereb Blood Flow Metab Offic J Int Soc Cereb Blood Flow Metabol 2003, 23(6):756-771.

92. Sengupta A, Mense SM, Lan C, Zhou M, Mauro RE, Kellerman L, Bentsman G, Volsky DJ, Louis ED, Graziano JH, et al: Gene expression profiling of human primary astrocytes exposed to manganese chloride indicates selective effects on several functions of the cells. NeuroToxicol 2007, 28(3):478-489.
93. Hazell AS, Norenberg MD: Ammonia and manganese increase arginine uptake in cultured astrocytes. Neurochem Res 1998, 23(6):869-873.

94. Tjalkens RB, Liu X, Mohl B, Wright T, Moreno JA, Carbone DL, Safe S: The peroxisome proliferator-activated receptor-gamma agonist 1, 1-bis(3'-indolyl)-1-(p-trifluoromethylphenyl)methane suppresses manganese-induced production of nitric oxide in astrocytes and inhibits apoptosis in cocultured PC12 cells. J Neurosci Res 2008, 86(3):618-629.

95. Barhoumi R, Faske J, Liu X, Tjalkens RB: Manganese potentiates lipopolysaccharide-induced expression of NOS2 in C6 glioma cells through mitochondrial-dependent activation of nuclear factor kappaB. Brain Res Mol Brain Res 2004, 122(2):167-179.

96. Rama Rao KV, Jayakumar AR, Tong X, Curtis KM, Norenberg MD: Brain aquaporin-4 in experimental acute liver failure. J Neuropathol Exp Neurol 2010, 69(9):869-879.

97. Giordano G, Pizzurro D, VanDeMark K, Guizzetti M, Costa LG: Manganese inhibits the ability of astrocytes to promote neuronal differentiation. Toxicol Appl Pharmacol 2009, 240(2):226-235

98. Broer S, Brookes N: Transfer of glutamine between astrocytes and neurons. J Neurochem 2001, 77(3):705-719.

99. Chaudhry FA, Krizaj D, Larsson P, Reimer RJ, Wreden C, Storm-Mathisen J, Copenhagen D, Kavanaugh M, Edwards RH: Coupled and uncoupled proton movement by amino acid transport system N. EMBO J 2001, 20(24):7041-7051.

100. Chaudhry FA, Reimer RJ, Edwards RH: The glutamine commute: take the $\mathrm{N}$ line and transfer to the A. J Cell Biol 2002, 157(3):349-355.

101. Broer A, Brookes N, Ganapathy V, Dimmer KS, Wagner CA, Lang F, Broer S: The astroglial ASCT2 amino acid transporter as a mediator of glutamine efflux. J Neurochem 1999, 73(5):2184-2194.

102. Broer $A$, Wagner $C A$, Lang F, Broer S: The heterodimeric amino acid transporter 4F2hc/y+LAT2 mediates arginine efflux in exchange with glutamine. Biochem J 2000, 349(Pt 3):787-795.

103. Xiang J, Ennis SR, Abdelkarim GE, Fujisawa M, Kawai N, Keep RF: Glutamine transport at the blood-brain and blood-cerebrospinal fluid barriers. Neurochem Int 2003, 43(4-5):279-288.

104. Sidoryk-Wegrzynowicz M, Lee E, Albrecht J, Aschner M: Manganese disrupts astrocyte glutamine transporter expression and function. J Neurochem 2009, 110(3):822-830.

105. Sidoryk-Wegrzynowicz M, Lee ES, Ni M, Aschner M: Manganese-induced downregulation of astroglial glutamine transporter SNAT3 involves ubiquitin-mediated proteolytic system. Glia 2010, 58(16):1905-1912.

106. Balkrishna S, Broer A, Kingsland A, Broer S: Rapid downregulation of the rat glutamine transporter SNAT3 by a caveolin-dependent trafficking mechanism in Xenopus laevis oocytes. Am J Physiol Cell Physiol 2010 299(5):C1047-1057.

107. Pawlik TM, Souba WW, Sweeney TJ, Bode BP: Phorbol esters rapidly attenuate glutamine uptake and growth in human colon carcinoma cells. J Surg Res 2000, 90(2):149-155.

108. Nissen-Meyer LS, Popescu MC, Chaudhry FA, Hamdani el H: Protein kinase C-mediated phosphorylation of a single serine residue on the rat glial glutamine transporter SN1 governs its membrane trafficking. J Neurosci 2011, 31(17):6565-6575.

109. Jentsch S, Schlenker S: Selective protein degradation: a journey's end within the proteasome. Cell 1995, 82(6):881-884.

110. Geetha T, Wooten MW: TrkA receptor endolysosomal degradation is both ubiquitin and proteasome dependent. Traffic 2008, 9(7):1146-1156.

111. Rotin D, Staub O: Nedd4-2 and the regulation of epithelial sodium transport. Front Physiol 2012, 3:212.

112. Boehmer C, Okur F, Setiawan I, Broer S, Lang F: Properties and regulation of glutamine transporter SN1 by protein kinases SGK and PKB. Biochem Biophys Res Commun 2003, 306(1):156-162.

113. Guilarte TR, Burton NC, Verina T, Prabhu W, Becker KG, Syversen T Schneider JS: Increased APLP1 expression and neurodegeneration in the frontal cortex of manganese-exposed non-human primates. J Neurochem 2008, 105(5):1948-1959.

114. Miranda M, Dionne KR, Sorkina T, Sorkin A: Three ubiquitin conjugation sites in the amino terminus of the dopamine transporter mediate protein kinase C-dependent endocytosis of the transporter. Mol Biol Cell 2007, 18(1):313-323.

115. Sorkina T, Miranda M, Dionne KR, Hoover BR, Zahniser NR, Sorkin A: RNA interference screen reveals an essential role of Nedd4-2 in dopamine transporter ubiquitination and endocytosis. J Neurosci Offic J Soc Neurosci 2006, 26(31):8195-8205. 
116. Mayer ML, Westbrook GL: The physiology of excitatory amino acids in the vertebrate central nervous system. Prog Neurobiol 1987, 28(3):197-276.

117. Tanaka K, Watase K, Manabe T, Yamada K, Watanabe M, Takahashi K, Iwama $H$, Nishikawa T, Ichihara N, Kikuchi T, et al: Epilepsy and exacerbation of brain injury in mice lacking the glutamate transporter GLT-1. Science 1997, 276(5319):1699-1702.

118. Watase K, Hashimoto K, Kano M, Yamada K, Watanabe M, Inoue Y, Okuyama S, Sakagawa T, Ogawa S, Kawashima N, et al: Motor discoordination and increased susceptibility to cerebellar injury in GLAST mutant mice. Eur J Neurosci 1998, 10(3):976-988.

119. Sheldon AL, Robinson MB: The role of glutamate transporters in neurodegenerative diseases and potential opportunities for intervention. Neurochem Int 2007, 51(6-7):333-355.

120. Lee ES, Sidoryk M, Jiang H, Yin Z, Aschner M: Estrogen and tamoxifen reverse manganese-induced glutamate transporter impairment in astrocytes. J Neurochem 2009, 110(2):530-544.

121. Mutkus L, Aschner JL, Fitsanakis V, Aschner M: The in vitro uptake of glutamate in GLAST and GLT-1 transfected mutant CHO-K1 cells is inhibited by manganese. Biol Trace Elem Res 2005, 107(3):221-230.

122. Sidoryk-Wegrzynowicz M, Lee E, Aschner M: Mechanism of Mn(II)-mediated dysregulation of glutamine-glutamate cycle: focus on glutamate turnover. J Neurochem 2012, 122(4):856-867.

123. Susarla BT, Robinson MB: Internalization and degradation of the glutamate transporter GLT-1 in response to phorbol ester. Neurochem Int 2008, 52(4-5):709-722.

124. Sidoryk-Wegrzynowicz M, Lee E, Albrecht J, Aschner M: Manganese disrupts astrocyte glutamine transporter expression and function. J Neurochem 2012, 110(3):822-830.

125. Conradt M, Stoffel W: Inhibition of the high-affinity brain glutamate transporter GLAST-1 via direct phosphorylation. J Neurochem 1997, 68(3):1244-1251

126. Kanthasamy AG, Kitazawa M, Kanthasamy A, Anantharam V: Role of proteolytic activation of protein kinase Cdelta in oxidative stressinduced apoptosis. Antioxid Redox Signal 2003, 5(5):609-620.

127. Latchoumycandane C, Anantharam V, Kitazawa M, Yang Y, Kanthasamy A, Kanthasamy AG: Protein kinase Cdelta is a key downstream mediator of manganese-induced apoptosis in dopaminergic neuronal cells. J Pharmacol Exp Ther 2005, 313(1):46-55.

128. Oubrahim H, Chock PB, Stadtman ER: Manganese(II) induces apoptotic cell death in NIH3T3 cells via a caspase-12-dependent pathway. J Biol Chem 2002, 277(23):20135-20138.

129. Chun HS, Lee H, Son JH: Manganese induces endoplasmic reticulum (ER) stress and activates multiple caspases in nigral dopaminergic neuronal cells, SN4741. Neurosci Lett 2001, 316(1):5-8.

130. Kitazawa M, Anantharam V, Yang Y, Hirata Y, Kanthasamy A, Kanthasamy AG: Activation of protein kinase $C$ delta by proteolytic cleavage contributes to manganese-induced apoptosis in dopaminergic cells: protective role of Bcl-2. Biochem Pharmacol 2005, 69(1):133-146.

doi:10.1186/2050-6511-14-23

Cite this article as: Sidoryk-Wegrzynowicz and Aschner: Role of astrocytes in manganese mediated neurotoxicity. BMC Pharmacology and Toxicology 2013 14:23.

\section{Submit your next manuscript to BioMed Central and take full advantage of:}

- Convenient online submission

- Thorough peer review

- No space constraints or color figure charges

- Immediate publication on acceptance

- Inclusion in PubMed, CAS, Scopus and Google Scholar

- Research which is freely available for redistribution 